\title{
Les médias et les médias dans la psychothérapie et la science de la psychothérapie
}

\section{Gerhard Burda}

\author{
Psychotherapie-Wissenschaft 10 (2) 952020 \\ www.psychotherapie-wissenschaft.info \\ CC BY-NC-ND \\ https://doi.org/10.30820/1664-9583-2020-2-95
}

Mots clés : Médium, différence de soi, psychothérapie, science de la psychothérapie, scepticisme radical

Le texte réunit un essai et un document de position sur la science de la psychothérapie (PTW).

L'essai compare deux approches différentes du processus thérapeutique : une qui se concentre sur des phénomènes concrets ou sur des faits objectivement donnés (médiateurs), et une seconde qui est orientée vers le processus spontané. La première se concentre sur certains phénomènes, symptômes ou quelque chose d'objectivement donnée qui peut être découverte " archéologiquement » ou explorée herméneutiquement. Cette approche présuppose des variables traditionnelles telles que le sujet, l'objet, l'identité, l'intersubjectivité, le soi, etc. L'ontologie qui la sous-tend est essentialiste et surtout elle cache les deux catégories de relation et d'altérité. La seconde se concentre davantage sur le processus spontané dans lequel tout être peut être compris comme un médium autodifférencié au sens ontologique. Le sujet, l'identité, le moi, etc. se forment constamment dans ce processus comme des interférences et ne doivent pas être considérés de manière isolée les uns des autres ; la base ontologique est, par rapport à la première approche, non pas rigide, mais " fluide ", fragile et passagère (Burda, 2011). L'idée de base est que les médias se médiatisent mutuellement dans le processus (médiamorphose), c'est-à-dire qu'ils sont modifiés en permanence par d'autres médias et ont un effet tout aussi changeant sur eux. Les médias sont dans une relation permanente de connexion et de séparation avec eux-mêmes ainsi qu'avec tout le reste. On montre dans quelle mesure l'être humain, la conscience et tout processus de cognition peuvent être compris en termes de constitution médiale. La psychothérapie (PT) devient ainsi une phénoménologie particulière de la guérison et de la recherche.
Cela sera expliqué au moyen de trois exemples, qui montrent que le processus de changement englobe des aspects inter- et intrapsychiques. Ces exemples pratiques illustrent comment le processus thérapeutique oscille constamment entre l'aspect le plus rigide et le plus dynamique et ce que cela peut signifier pour le travail thérapeutique sur les identifications. Il est également proposé d'utiliser le terme " média » comme parenthèse pour toutes les directions thérapeutiques.

Le document de position va plus loin dans cette idée en direction de la science de la psychothérapie. Il aborde la relation entre la psychothérapie et la science de la psychothérapie dans le contexte de l'épistémologie et de la philosophie des sciences. L'importance d'une perspective radicalement sceptique est soulignée, ce qui peut permettre de formuler une méta-théorie qui garantisse la nécessaire distance de la psychothérapie par rapport à elle-même et ouvre ainsi le champ de ses recherches psychothérapeutiques. Enfin, une brève explication est donnée sur la manière dont les termes " médiat " et « média » peuvent être utilisés méthodiquement.

\section{L'auteur}

Gerhard Burda est un philosophe, un scientifique en psychothérapie et un analyste de formation de la Société autrichienne de psychologie analytique.

\section{Contact}

PD Mag. DDr. Gerhard Burda

Penzinger Str. 69/11

1140 Wien, Austria

E-Mail : comger@gmx.at 